\title{
Enhancing photoluminescence quenching and photoelectric properties of CdSe quantum dots with hole accepting ligands $\dagger$
}

\author{
I-Shuo Liu, ${ }^{a}$ Hsi-Hsing Lo, ${ }^{a}$ Chih-Tao Chien, ${ }^{a}$ Yun-Yue Lin, ${ }^{a}$ Chun-Wei Chen, ${ }^{a}$ \\ Yang-Fang Chen, ${ }^{* b}$ Wei-Fang Su${ }^{* a}$ and Sz-Chian Liou ${ }^{c}$
}

\author{
Received 3rd October 2007, Accepted 10th December 2007 \\ First published as an Advance Article on the web 3rd January 2008 \\ DOI: $10.1039 / \mathrm{b} 715253 \mathrm{a}$
}

\begin{abstract}
CdSe quantum dots have been encapped with aromatic ligands: $\alpha$-toluenethiol, thiophenol, and p-hydroxythiophenol to enhance the photoluminescence (PL) quenching and photoelectric properties of the quantum dots. The aromatic ligand capped CdSe quantum dots are prepared through ligand exchange with trioctylphosphine oxide (TOPO) capped CdSe quantum dots. The XPS surface chemistry analysis and elemental analysis has confirmed the success of ligand exchange from TOPO to aromatic ligands. Both XRD and HRTEM-SAED studies indicate the crystalline structure of CdSe quantum dots not only remains but is also improved by the ligand exchange of TOPO with thiol molecules. Time resolved PL decay measurements indicate thiophenol and $p$-hydroxythiophenol ligands effectively quench the emission and have much shorter PL lifetimes than that of TOPO and that of $\alpha$-toluenethiol. Thus, both thiophenol and $p$-hydroxythiophenol can act as an effective acceptor for photogenerated holes through aromatic $\pi$-electrons. Thiophenol also exhibits good charge transport behavior showing a 10-fold increase in short circuit current density $\left(I_{\mathrm{sc}}\right)$ as compared with TOPO in the photocurrent study of fabricated photovoltaic devices.
\end{abstract}

\section{Introduction}

Research concerning the size-dependent optical properties of colloidal semiconductor quantum dots has had increasing interest during the past decade because of their applications in photostable luminescent biological labels, ${ }^{1-3}$ solar cells, ${ }^{4-12}$ and lightemitting devices. ${ }^{13-16}$ The quantum dots are usually surrounded by a ligand capping layer to prevent their coagulation. The nature of the ligand strongly affects the luminescent properties of quantum dots. ${ }^{17}$ Synthesized CdSe nanocrystals are covered with organic ligands containing bulky (C8-C18) alkyl groups attached through the classic organometallic route ${ }^{18}$ and numerous "greener" modifications. ${ }^{19-27} \mathrm{CdSe}$ nanocrystals that are coated with inorganic materials, such as $\mathrm{CdS}, \mathrm{ZnS}$ or $\mathrm{ZnSe}$ layers for biological labeling applications, can improve the fluorescence quantum yield ${ }^{28}$ and, in the case of $\mathrm{ZnS}$ or $\mathrm{ZnSe}$, decrease the toxicity of the quantum dot. The use of a shell with higher bandgap materials such as $\mathrm{CdS}, \mathrm{ZnS}, \mathrm{ZnSe}$, or long chain bulky alkyl group ligands (acting as a potential barrier) is not suitable for applications involving charge transfer. Almost all common and readily accessible surfactants are alkyl thiols, ${ }^{19,29,30}$ pyridine, ${ }^{31}$ amines, ${ }^{19,26}$ phosphonic acid,,$^{32}$ and phosphine oxides, ${ }^{33}$ most of which are

\footnotetext{
${ }^{a}$ Department of Materials and Engineering, National Taiwan University, Taipei, Taiwan. E-mail: suwf@ntu.edu.tw; Fax: +886-2-33664078; Tel: $+886-2-33664078$

${ }^{b}$ Department of Physics, National Taiwan University, Taipei, Taiwan. E-mail: yfchen@phys.ntu.edu.tw; Tel: +886-2-33665125

${ }^{c}$ Center for Condensed Matter Sciences, National Taiwan University, Taipei, Taiwan. E-mail: basieliou@yahoo.com.tw; Tel: +886-2-33665210 $\uparrow$ Electronic supplementary information (ESI) available: Details of crystalline structure of CdSe quantum dots after ligand exchange of TOPO with thiol molecules. See DOI: 10.1039/b715253a
}

electrical insulators with large bandgaps. In many examples, it is desirable to facilitate electron transfer access to the surface, while simultaneously controlling the dispersion or solubility of nanocrystals. ${ }^{32}$ Earlier work has provided an interesting example of surfactant facilitated electronic interaction, where conduction through a Au nanocrystal film was mediated by conjugated thiols with resonant energy levels, which displaced insulating alkyl thiols. ${ }^{34}$

Solar cells based on semiconductor nanocrystals are promising to be a new approach to improve the efficiency of photovoltaic devices. ${ }^{4-12}$ They also show great promise for CdSe nanocrystals of different shapes and sizes as active components of light emitting diodes ${ }^{13-16}$ and photovoltaic cells. ${ }^{4-12}$ Charge transfer at the organic/inorganic interface has been found to be enhanced because the inorganic semiconductors have greater electron affinities which are energetically favorable for the electron to transfer onto the organic materials. One problem that limits the efficiency of these devices is the bulky or insulating organic layer of the CdSe nanocrystals. Typically, the hydrophobic surface capping agents (usually consisting of long alkyl chain components) will hinder charge transfer. ${ }^{35} \mathrm{~A} 1.1 \mathrm{~nm}$ thick monolayer of the TOPO surfactant on the surface of CdSe nanocrystals was sufficient to reduce electron transfer efficiency by a factor of 10 in the case of CdSe and poly(2-methoxy-5-(2'ethylhexyloxy)- $p$-phenylenevinylene) (MEHPPV). ${ }^{4}$ Thus, there is a significant need for a surfactant that can be controlled to disperse the semiconductor nanocrystals in a variety of solvents or polymers, while also permitting electron and hole transfer between nanocrystals and organic semiconductors. CdSe nanocrystals coated with an electroactive surfactant designed to facilitate the charge transfer quickly between surroundings have been studied. ${ }^{32}$ Some groups tried to wash out the bulky surfactants to 
form bare nanocrystals and they found that TOPO capped CdSe nanocrystals can be replaced with a hydrophilic moiety such as pyridine then dispersed into a conjugated polymer matrix. ${ }^{6,35}$ Pyridine capped CdSe nanocrystal/conjugated polymer nanocomposites have been investigated extensively for their applications in optoelectronics and photovoltaic devices. ${ }^{6}$

Despite the importance of surface exchange reactions with organic ligands for the application of semiconductor nanocrystals, the surfaces of nanocrystals are poorly understood. This is especially true with regard to how the organic capping layer influences the optical and photoelectric properties of CdSe nanocrystals. To our knowledge, most researchers studied the ligand effects on the optical properties of CdSe nanocrystals, ${ }^{36-46}$ and comparison of the photoelectric properties of different ligandcapped CdSe nanocrystals was rare. ${ }^{39}$ In this paper, we examine hole transfer from photoexcited CdSe quantum dots to hole accepting ligands: thiophenol and $p$-hydroxythiophenol and compare the results with none-hole accepting ligands: TOPO and $\alpha$-toluenethiol. We analyzed these systems with PL spectra and time-resolved PL decay spectra. Our results suggest that thiophenol and p-hydroxythiophenol transfer the photogenerated hole in CdSe very effectively. The results of current-voltage $(I-V)$ measurements showed the short-circuit current density of thiophenol capped CdSe quantum dots to be an order higher than that of TOPO capped CdSe quantum dots.

\section{Experimental}

The materials used were all of the purest quality available and used as received. CdSe nanocrystals were prepared using the method described by Peng and Peng. ${ }^{21}$ Briefly, CdO (0. $41 \mathrm{~g}$, 99.99\%, Aldrich), trioctylphosphine oxide (TOPO, $61 \mathrm{~g}, 90 \%$, Aldrich), and hexadecylamine (HDA, 30.5 g, 90\%, Tokyo Kasei) were loaded into a $250 \mathrm{ml}$ flask. The mixture was then heated to about $320^{\circ} \mathrm{C}$ under Ar flow with stirring. Once the color of the solution changed from brick red to transparent, the temperature of the solution was cooled to $240{ }^{\circ} \mathrm{C}$ and a solution of Se powder $(0.25 \mathrm{~g}, 99 \%$, Aldrich) dissolved in tri-n-butylphosphine (TBP, 5 $\mathrm{ml} \mathrm{95 \% ,Acros)} \mathrm{was} \mathrm{quickly} \mathrm{injected} \mathrm{into} \mathrm{the} \mathrm{flask.} \mathrm{After} \mathrm{the}$ injection, the CdSe quantum dots were allowed to age at $240{ }^{\circ} \mathrm{C}$ for $1 \mathrm{~min}$ before cooling to room temperature under Ar atmosphere. CdSe quantum dots were isolated by adding $15 \mathrm{ml}$ of methanol (99.99\%, Acros) to the cooled reaction vessel followed by centrifugation. Repeated methanol washings followed by centrifugation removed excess TOPO and obtained TOPO passivated CdSe quantum dot powder. This preparation typically yielded $\sim 3 \mathrm{~nm}$ diameter CdSe quantum dots.

To modify the surface of CdSe quantum dots with different organic ligands, we employed a ligand exchange procedure. The TOPO ligand on the surface of the CdSe quantum dots was removed by repeat pyridine washing. The CdSe quantum dot powder containing TOPO was re-dispersed in pyridine (99\%, Acros) to remove TOPO. Washed CdSe quantum dots were precipitated by adding excess hexane $(99 \%$, Fluka) and then isolated by centrifugation. This exchange procedure was repeated 6 times. Most of the TOPO molecules on the surface of the CdSe quantum dots are exchanged by pyridine. To remove the surface pyridine, CdSe quantum dots were kept in vacuum overnight.
To passivate the surface of the quantum dot with thiophenol (99\%, Acros) or $\alpha$-toluenethiol (99\%, Acros), bare CdSe quantum dots $(0.2 \mathrm{~g})$ were re-dispersed in a mixture of $25 \mathrm{~g}$ benzene $(99 \%$, Fluka) and 0.1 mole of either thiophenol or $\alpha$-toluenethiol. The solution was left to stir for 1 day in Ar flow at $70{ }^{\circ} \mathrm{C}$. At the end of the exchange reaction, the nanocrystals were recovered as a precipitate by adding an excess of hexane to the solution. This ligand exchange cycle was repeated three times. Finally, the CdSe quantum dots passivated with thiophenol or $\alpha$-toluenethiol were stored in Ar environment at $-10{ }^{\circ} \mathrm{C}$.

For $p$-hydroxythiophenol capped quantum dots, bare $\mathrm{CdSe}$ nanocrystals $(0.2 \mathrm{~g})$ were re-dispersed in a mixture of $25 \mathrm{~g}$ methanol and $0.3 \mathrm{~g} p$-hydroxythiophenol (99\%, Tokyo Kasei). The reaction was left to stir for 1 day in Ar environment at 70 ${ }^{\circ} \mathrm{C}$. After the exchange reaction, nanocrystals were precipitated by excess hexane. We repeated the above ligand exchange cycle three times. The nanocrystals passivated with $p$-hydroxythiophenol were also stored in $\mathrm{Ar}$ atmosphere at $-10{ }^{\circ} \mathrm{C}$. The yield of the ligand exchange process was approximately $40 \%$. The relatively low yield is due to the initial TOPO-CdSe quantum dots containing an excess amount of TOPO.

X-Ray photoelectron spectra (XPS) were recorded by using an electron spectroscope for chemical analysis system (VG Scientific ESCALAB 250). The experiments were performed under ultrahigh vacuum. Both $\mathrm{Mg}$ and $\mathrm{Al}$ anodes driven at $400 \mathrm{~W}$ were used for this study. All scans were obtained using the $0.5 \mathrm{~cm}^{2}$ aperture. Typical surveys were collected for $5 \mathrm{~min}$.

The amounts of $\mathrm{C}, \mathrm{H}, \mathrm{N}$, and $\mathrm{S}$ atoms were measured by using a Perkin-Elmer 2400 Heraeus varioIII-NCSH for elemental analysis.

The crystalline structure of the quantum dots was studied using X-ray diffraction (XRD) (Philips PW3040) with filtered $\mathrm{Cu} \mathrm{K} \alpha$ radiation $(\lambda=1.54056 \AA)$ and a JOEL JEM-1230 high resolution transmission electron microscope (HRTEM) at $200 \mathrm{keV}$.

UV-Visible absorption spectra were obtained using a PerkinElmer Lambda 35 UV/VIS Spectrometer. The steady-state PL spectra were measured by using a Perkin-Elmer FS-55 spectrofluorometer. Time-resolved PL spectroscopy was performed with a time-correlated single photon counting (TCSPC) spectrometer (Picoquant, Inc.). A pulse laser $(375 \mathrm{~nm})$ with an average power of $1 \mathrm{~mW}$ operating at $40 \mathrm{MHz}$ with a duration of 70 ps was used for excitation.

Photovoltaic devices were fabricated using a sandwich structure of ITO/PEDOT: PSS (Aldrich)/CdSe quantum dots/ Al. The PEDOT:PSS was spin coated onto a pre-cleaned, patterned ITO substrate and was subsequently dried at $120^{\circ} \mathrm{C}$. The CdSe quantum dots were dissolved in chlorobenzene and then spin coated on top of the dried PEDOT:PSS. The thickness of the PEDOT: PSS layer was kept at about $100 \mathrm{~nm}$. The thicknesses of the TOPO capped CdSe quantum dot, $\alpha$-toluenethiol capped CdSe quantum dot, and thiophenol capped CdSe quantum dot layers were $47 \mathrm{~nm}, 51 \mathrm{~nm}$, and $55 \mathrm{~nm}$ respectively. The aluminium electrode layer was thermal evaporated onto the active layer and the electrode layer was kept at a thickness of $130 \mathrm{~nm}$. The current-voltage characterization $(I-V)$ (Keithley 2400 sourcemeter) was performed under vacuum, at $10^{-3}$ Torr, with monochromatic illumination at a defined beam size. 


\section{Results and discussion}

High-resolution XPS spectra were used to study the surface chemistry of ligand encapped CdSe nanoparticles (Fig. 1). The TOPO capped CdSe quantum dot has the $\mathrm{Cd} 3 \mathrm{~d} 5 / 2$ peak in the range from 405 to $406 \mathrm{eV}$, with a normal distribution centered at $405.6 \mathrm{eV}$ which agrees with previously reported data ${ }^{47}$ of 405.6 $\pm 0.2 \mathrm{eV}$ (Fig. 1(a)); the Se $3 \mathrm{~d}$ peak ranged from 53 to $56 \mathrm{eV}$, with a normal distribution centered at $54.4 \mathrm{eV}$ which also agrees with the literature data ${ }^{47}$ of $54.6 \pm 0.2 \mathrm{eV}$ (Fig. 1(b)). The Cd 3d5/2

(a)

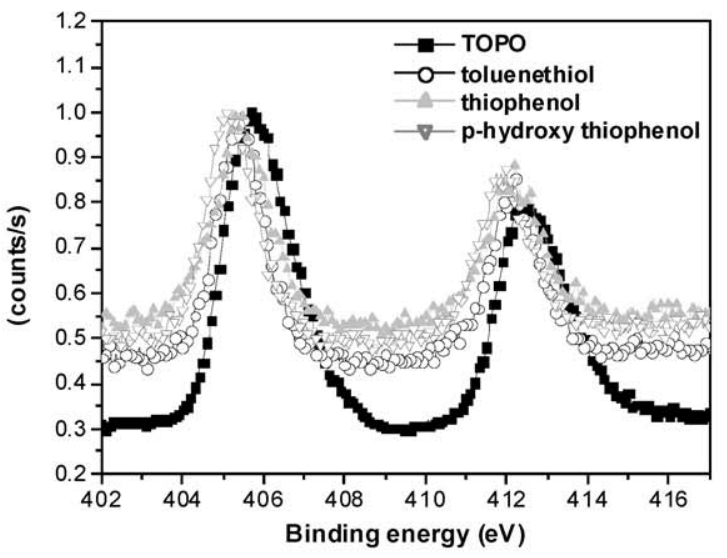

(b)

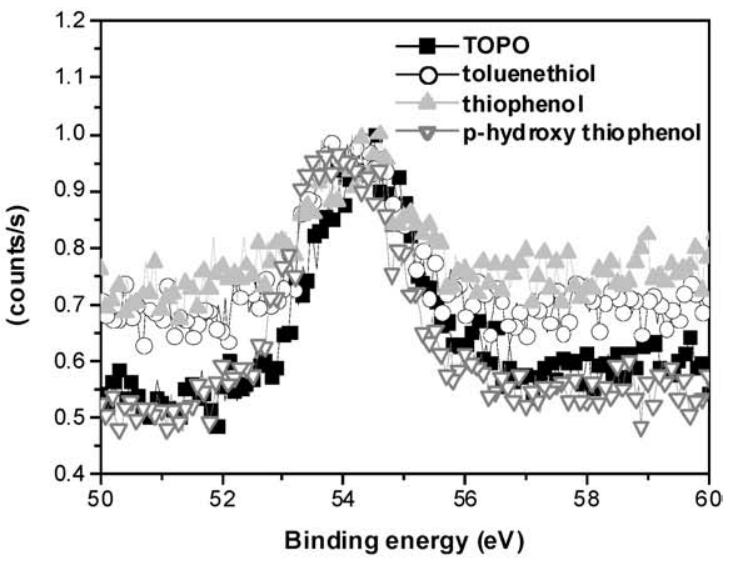

(c)

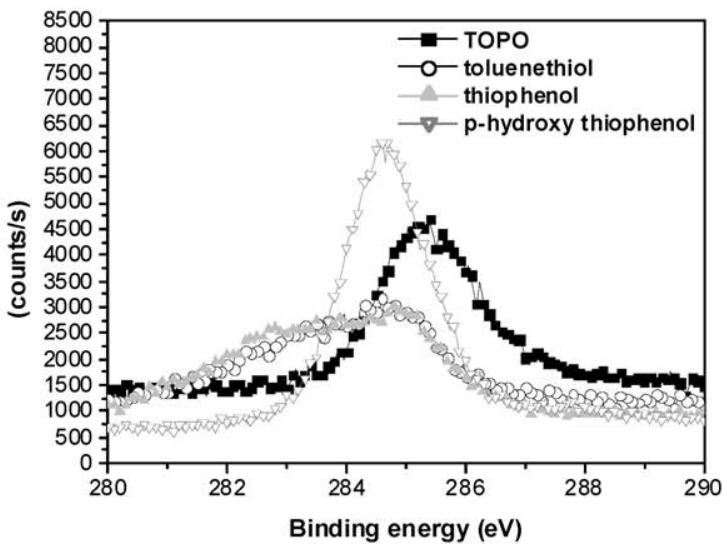

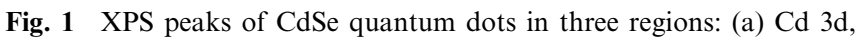
(b) Se 3d, and (c) C 1s. and $\mathrm{Cd} 3 \mathrm{~d} 3 / 2$ XPS spectra of different ligand capped CdSe quantum dots ( $\alpha$-toluenethiol, thiophenol, and $p$-hydroxythiophenol) deviate from those of the TOPO capped CdSe quantum dots. This implies their surface states are different from those of the TOPO capped CdSe quantum dots. However, their Se 3d peak positions rarely deviate from those of the TOPO capped CdSe quantum dots. It has been reported that the thiol functional groups of ligands will complex with the $\mathrm{Cd}$ atoms of the $\mathrm{CdSe}$ surfaces to form $\mathrm{Cd}-\mathrm{S}$ structures. ${ }^{19,29-30}$ Puzder et al. calculated the binding energy of phosphine oxide to the CdSe quantum dot facets. ${ }^{48,49}$ They found that the dominant binding interaction is between oxygen atoms in the ligands and cadmium atoms on the quantum dot surfaces. Our results imply the ligands TOPO, thiophenol, $\alpha$-toluenethiol, and $p$-hydroxythiophenol bind much stronger to the $\mathrm{Cd}$ atoms than to the Se atoms of the CdSe quantum dots. Oxide peaks as a doublet near $55 \mathrm{eV}$ for $\mathrm{Se} 3 \mathrm{~d}^{50}$ and $405 \mathrm{eV}$ for $\mathrm{Cd} 3 \mathrm{~d}^{47}$ were not observed for exchanged ligands on CdSe quantum dots. This indicates that the ligand exchange on the quantum dots is successful and the exchanged quantum dots are in good quality. In order to check that the exchanged ligands really bond on the surfaces of $\mathrm{CdSe}$ quantum dots, XPS spectra of the $\mathrm{C}$ region were taken. The $\mathrm{C}$ atoms of saturated aliphatic TOPO were monitored for the C 1s peak position and found to range from 284 to $287 \mathrm{eV}$, with a normal distribution centered about $285.3 \mathrm{eV} .^{51}$ The $\mathrm{C}$ atoms of aromatic ligands were monitored for the $\mathrm{C} 1 \mathrm{~s}$ peak positions of thiophenol, $\alpha$-toluenethiol, and $p$-hydroxythiophenol, and found to center about $284.7 \mathrm{eV} .^{52}$ These results imply that the thiophenol, $\alpha$-toluenethiol, and $p$-hydroxythiophenol were successfully capped on the surfaces of CdSe quantum dots. The $282.8 \mathrm{eV}$ peak positions of thiophenol and $\alpha$-toluenethiol represent the $\mathrm{C}-\mathrm{Si}$ bond $^{53}$ from using a silicon substrate in the XPS experiment.

The FTIR spectra of the TOPO capped CdSe quantum dots, thiophenol capped CdSe quantum dots, $\boldsymbol{\alpha}$-toluenethiol capped CdSe quantum dots, and $p$-hydroxythiophenol capped $\mathrm{CdSe}$ quantum dots are presented in Fig. 2. The $\mathrm{OH}$ vibration peak at $3430 \mathrm{~cm}^{-1}$ comes from the water. Water adsorbs on the surfaces of CdSe quantum dots because of its high surface-tovolume ratio. From Fig. 2(a), the $\mathrm{P}=\mathrm{O}$ stretch of the TOPO

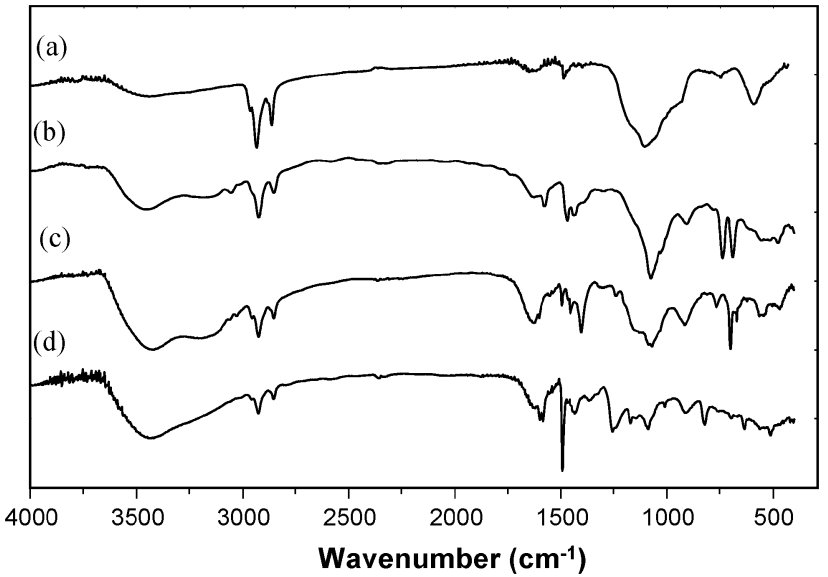

Fig. 2 FTIR spectra of (a) TOPO capped, (b) thiophenol capped, (c) $\alpha$-toluenethiol capped, and (d) $p$-hydroxythiophenol capped CdSe quantum dots. 
capped CdSe quantum dots at $1082 \mathrm{~cm}^{-1}$ is in agreement with the literature. ${ }^{47}$ The splitting with a shift to lower energy of the band could imply some multidentate coordination through occupation of bridging positions over the Cd sites. ${ }^{47}$ The bands at around $2925 \mathrm{~cm}^{-1}$ and $2850 \mathrm{~cm}^{-1}$ in Fig. 2(a) to (d) can be assigned as a $\mathrm{C}-\mathrm{C}-\mathrm{H}$ stretching that implies there are still small amounts of TOPO on the CdSe quantum dots after ligand exchange. There is a band at around $3055 \mathrm{~cm}^{-1}$ in Fig. 2(b) to (d) which can be interpreted as the aromatic $\mathrm{C}=\mathrm{C}-\mathrm{H}$ stretching. This implies that the ligands (thiophenol, $\alpha$-toluenethiol, and p-hydroxythiophenol) cap on the CdSe quantum dot surfaces. The three peaks between $1750 \mathrm{~cm}^{-1}$ and $1250 \mathrm{~cm}^{-1}$ (thiophenol: $1575 \mathrm{~cm}^{-1}, 1469 \mathrm{~cm}^{-1}$, and $1433 \mathrm{~cm}^{-1} ; \alpha$-toluenethiol: 1620 $\mathrm{cm}^{-1}, 1452 \mathrm{~cm}^{-1}$, and $1400 \mathrm{~cm}^{-1} ; p$-hydroxythiophenol: 1583 $\mathrm{cm}^{-1}, 1492 \mathrm{~cm}^{-1}$, and $1435 \mathrm{~cm}^{-1}$ ) in Fig. 2(b) to (e) come from the aromatic $\mathrm{C}=\mathrm{C}$ bending. The $1076 \mathrm{~cm}^{-1}$ peak in Fig. 2(b), the $1068 \mathrm{~cm}^{-1}$ peak in Fig. 2(c), and the $1081 \mathrm{~cm}^{-1}$ peak in Fig. 2(d) come from the remnants of TOPO. In Fig. 2(b) to (d), we do not observe the $\mathrm{S}-\mathrm{H}$ peak at about $2572 \mathrm{~cm}^{-1}$. This implies that the thiol bond breaks and the sulfur then bonds to the $\mathrm{Cd}$ atom, $-\mathrm{S}-\mathrm{Cd} .{ }^{54}$

To determine the amount of ligands per weight of CdSe quantum dots, elemental analysis was performed. Because there is only one $\mathrm{S}$ atom per each molecule of thiophenol, $\boldsymbol{\alpha}$-toluenethiol, and $p$-hydroxythiophenol respectively, we can determine the concentration of ligand by moles of $\mathrm{S}$ atoms per $100 \mathrm{~g} \mathrm{CdSe}$ quantum dots (Table 1). If we assume that the TOPO ligands on the surface of CdSe quantum dots were completely replaced by thiophenol, $\alpha$-toluenethiol, and $p$-hydroxythiophenol ligands respectively, then the amount of CdSe per mole of each ligand can be determined. Finally, the moles of $\mathrm{S}$ atoms for each ligand on the surface of CdSe quantum dots should be the same. Table 1 shows that the concentration of $\mathrm{S}$ atoms for each ligand is similar. This result also indicates the ligand exchange between TOPO and the other ligand is quite complete. The crystalline structures and morphologies of CdSe quantum dots with different ligands were studied by XRD and high-resolution transmission electron microscopy (HRTEM). The results show the crystalline structure of CdSe quantum dot not only remains but is also improved by the ligand exchange of TOPO with thiol molecules. The details are summarized and discussed in the ESI. $\dagger$

The optical properties of CdSe quantum dots with different ligands were studied by UV-Vis absorption spectroscopy and PL spectroscopy. To avoid thickness and geometry effects, the optical density of absorption and PL intensity were measured in solution within cuvette cells at the same quantum dot concentration. The samples were prepared by dissolving quantum dots at $0.05 \%$ by weight in chlorobenzene except for $p$-hydroxythiophenol capped CdSe which was dissolved in ethanol. The excitonic absorption peak at about $560 \mathrm{~nm}$ (Fig. 3) exhibits a blue shift with respect to that of the bulk CdSe due to the quantum

Table 1 Mole concentration of S atom per $100 \mathrm{~g}$ CdSe for different ligands

\begin{tabular}{ll}
\hline Ligand & Moles of S per $100 \mathrm{~g} \mathrm{CdSe}$ \\
\hline Thiophenol & 0.1489 \\
$\alpha$-Toluenethiol & 0.1339 \\
$p$-Hydroxythiophenol & 0.1373 \\
\hline
\end{tabular}

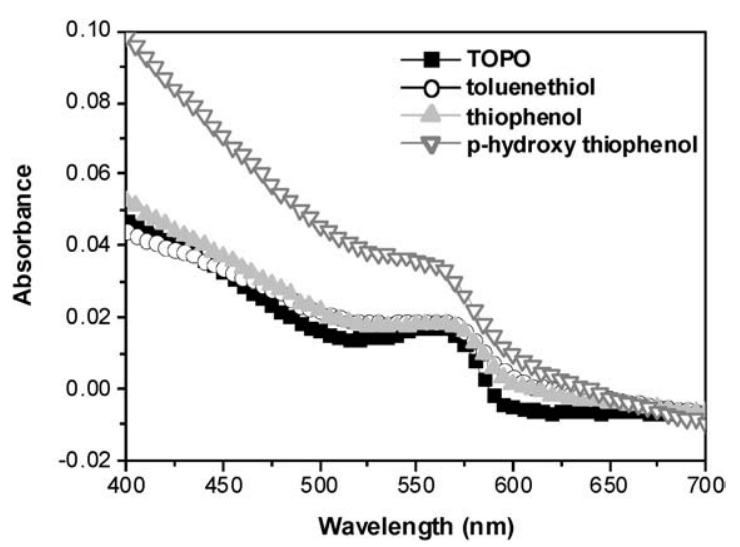

Fig. 3 Absorption spectra of CdSe quantum dots with different ligands.

confinement effect. The $p$-hydroxythiophenol capped CdSe quantum dots in ethanol showed a higher absorption than other ligand capped $\mathrm{CdSe}$ quantum dots in chlorobenzene, due to their different solubility behaviors. The total absorption of colloid particles consists of two contributions: absorption and scattering. The relatively poor solubility of hydrophilic $p$-hydroxythiophenol capped CdSe quantum dots results in greater scattering than the other quantum dots which results in greater absorption. The absorption intensities of CdSe quantum dots with different ligands remain similar, while the yield of the PL emission behaves significantly differently (Fig. 4). The PL intensity of $\mathrm{CdSe}$ quantum dots is affected by the type of ligand with a decreasing order of TOPO $>\alpha$-toluenethiol $>$ thiophenol $>$ $p$-hydroxythiophenol. From the research, the decrease of PL intensity of thiol capped CdSe quantum dots compared to TOPO capped CdSe quantum dots can be explained by the lower energetic position of the valence band for CdSe resulting in hole trapping of the photogenerated hole on the thiol molecule. ${ }^{46}$ As we compare thiophenol and $p$-hydroxythiophenol with $\alpha$-toluenethiol, PL quenching is observed. $p$-Hydroxythiophenol has the largest PL quenching effect. Since the aromatic rings in thiophenol and $p$-hydroxythiophenol are effective quenchers of CdSe emission, this suggests that they are capable of directly intercepting one of the charge carriers, thus disrupting the radiative recombination process as shown in eqn 1 and 2 .

$$
\mathrm{CdSe}+h v \rightarrow \mathrm{CdSe}(\mathrm{h}+\mathrm{e}) \rightarrow \mathrm{CdSe}+h v^{\prime}
$$

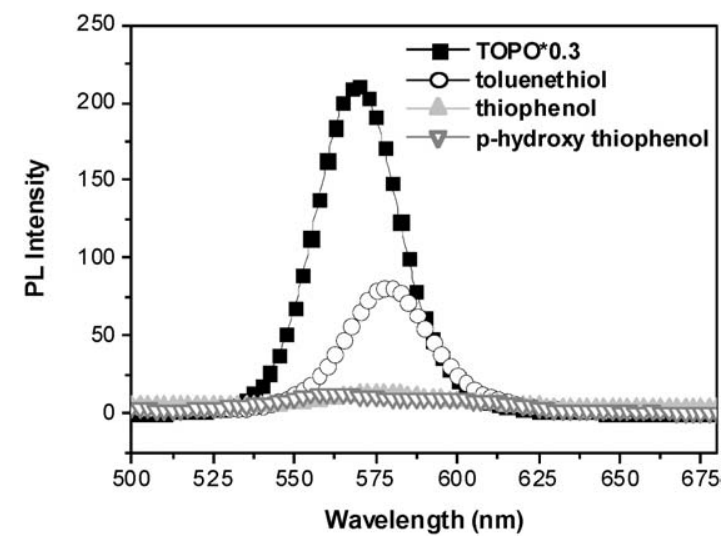

Fig. 4 PL spectra of CdSe quantum dots with different ligands. 


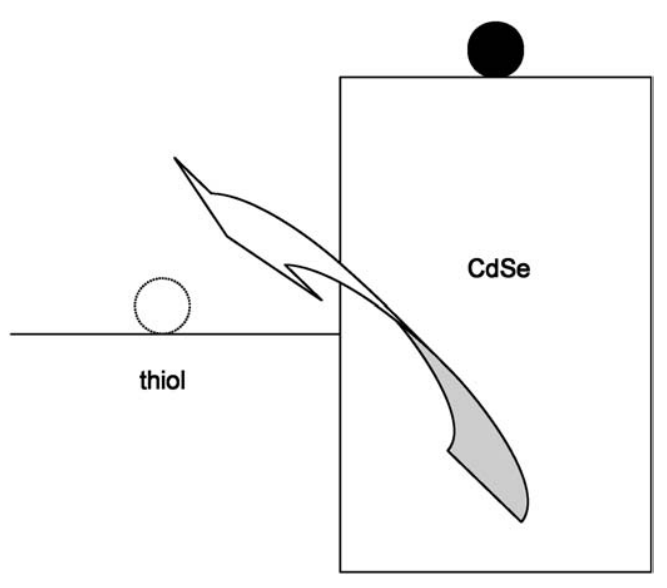

Fig. 5 A schematic diagram of the energy levels and charge separation of excitons at the surface of a thiol capped CdSe quantum dot.

$\mathrm{CdSe}(\mathrm{h})+$ thiophenol or $p$-hydroxythiophenol $\rightarrow$ $\mathrm{CdSe}+$ thiophenol $^{+}$or $p$-hydroxythiophenol ${ }^{+}$

Since the reduction potential of thiophenol is $-0.006 \mathrm{~V} v s$. NHE, ${ }^{55,56}$ it can effectively scavenge the photogenerated holes from the CdSe surface. The valence band edge of CdSe quantum dots is expected to be around $+1.2 \mathrm{~V} v s$. NHE. Both the size quantization effect and surface bound ligands are expected to shift the valence band to a more positive potential which will be a stronger oxidant than the bulk. This shift in valence band is likely to be $\leq 0.5 \mathrm{~V}$. Thus, from eqn (1), when the photon is absorbed by the CdSe quantum dot, an excited electron-hole pair exciton will form. The photogenerated hole in the CdSe quantum dot will transfer to the ligand, thiophenol or $p$-hydroxythiophenol (eqn (2)). The electron will be located in the CdSe region and the hole will be located in the thiol region as shown in Fig. 5. When the hole is trapped on the thiol ligands, radiative recombination of the exciton is not possible resulting in a strongly reduced PL intensity. If the valence band of the semiconductor nanocrystal is situated at lower energies than the thiol redox energy level, the hole trapping from the semiconductor nanocrystal to a thiol will be energetically favorable. CdSe can efficiently convert thiols into disulfides by a photocatalytic reaction. ${ }^{57}$ First, a thiol molecule that is strongly adsorbed at the CdSe surface will trap a photogenerated hole. Hole trapping on a thiol molecule results in the creation of a thiyl radical, and then two thiyl radicals can be converted into a disulfide.

We further probed the interaction between CdSe and ligands by monitoring the emission decay using a $375 \mathrm{~nm}$ laser pulse as the excitation source. The emission intensity recorded at the emission maximum (Fig. 6) exhibited a multiexponential decay and was analyzed using biexponential decay kinetics which can be expressed by eqn (3).

$$
F(t)=a_{1} \exp \left(-t / \tau_{1}\right)+a_{2} \exp \left(-t / \tau_{2}\right)
$$

The explanation for the origin of multiexponential emission decay of metal chalcogenides has been studied in detail from the trapping sites within the nanocrystal. ${ }^{58-63}$ Surface defects give rise to trap states that lie within the bandgap and complicate the emission dynamics. Excitation of CdSe promotes an electron

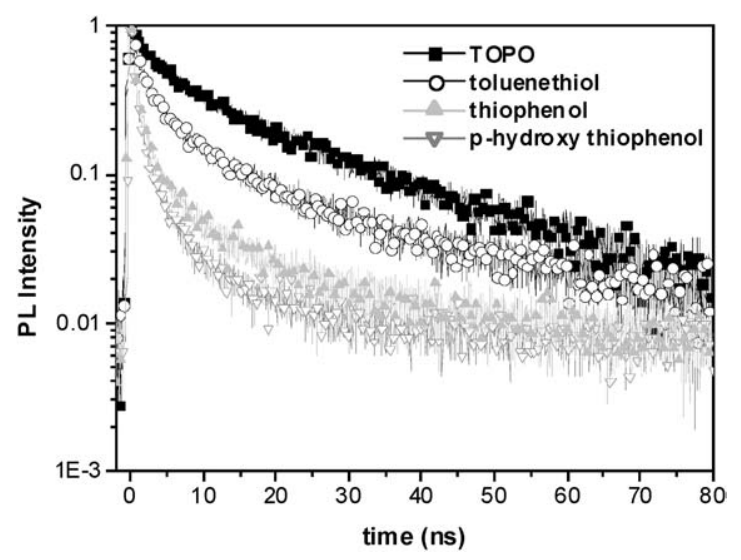

Fig. 6 PL lifetime decay of CdSe quantum dots with different ligands.

high in the conduction band, and then the excited electron relaxes quickly to the bottom of the conduction band. The exciton emission arises from radiative relaxation of these electrons to the ground state and contributes to the fastest lifetime decays. However, these conduction band electrons may be localized in shallow trap states. These electrons in shallow trap states may repopulate the conduction band or thermalize into deeper trap states. From the former case, the lifetime is extended; the latter contributes to nonradiative mechanisms. A combination of all of these processes along with differences between the individual nanocrystals in a population gives rise to multiexponential emission dynamics that occur over a nanosecond time scale and were observed in this experiment. For the TOPO ligand, the CdSe quantum dots exhibit a lifetime of $2.79 \mathrm{~ns}\left(a_{1}=\right.$ $0.402)$ and $16.73 \mathrm{~ns}\left(a_{2}=0.523\right)$, respectively. For the other three ligands, the emission lifetimes $\left(\tau_{1}\right.$ and $\left.\tau_{2}\right)$ were decreased. Of these, thiophenol and $p$-hydroxythiophenol showed the most significant decrease. Therefore, the charge separations at the interfaces between ligands and CdSe quantum dots for thiophenol and $p$-hydroxythiophenol are quicker than for TOPO and $\alpha$-toluenethiol interfaces. From eqn (2), photogenerated holes will transfer from quantum dot to thiophenol or $p$-hydroxythiophenol ligands very quickly. $\alpha$-Toluenethiol contains an insulating methylene linkage between the aromatic ring and the thiol. This linkage cannot transfer charge for excitons obtained from $\alpha$-toluenethiol capped CdSe quantum dots. However, the lifetime of $\alpha$-toluenethiol capped CdSe is shorter than that of TOPO capped CdSe. This implies that a small amount of charge separation is available through the tunnel effects of aromatic ring $\pi$-electrons in $\alpha$-toluenethiol which are unavailable in TOPO. These results are consistent with the reported phenomenon that the radiative lifetimes of thiol capped CdSe quantum dots are much smaller than those of TOPO capped CdSe quantum dots. ${ }^{46}$

To compare the emission lifetimes of the different ligand capped CdSe quantum dots, we determined their average lifetimes $(\langle t\rangle)$ using eqn (4).

$$
\langle t\rangle=\sum a_{i} \tau_{i}^{2} / \sum a_{i} \tau_{i}
$$

By substituting the values of $a_{1}, a_{2}, \tau_{1}$, and $\tau_{2}$ into eqn (4), we obtained the average lifetime of each quantum dot. The results are summarized in Table 2. The emission lifetimes of these three ligand capped CdSe quantum dots in ascending order 
Table 2 Emission lifetimes of CdSe quantum dots with different ligands

\begin{tabular}{llrl}
\hline Ligand & $\tau_{1}\left(a_{1}\right)$ & \multicolumn{1}{c}{$\tau_{2}\left(a_{2}\right)$} & Avg lifetime/ns \\
\hline TOPO & $2.79(0.402)$ & $18.36(0.523)$ & 16.73 \\
$\alpha$-Toluenethiol & $1.66(0.648)$ & $14.21(0.273)$ & 11.49 \\
Thiophenol & $0.81(0.822)$ & $8.51(0.143)$ & 5.79 \\
$p$-Hydroxythiophenol & $0.62(0.909)$ & $5.29(0.151)$ & 3.36 \\
\hline
\end{tabular}

are: $p$-hydroxythiophenol capped CdSe quantum dots $(3.36 \mathrm{~ns})<$ thiophenol capped CdSe quantum dots $(5.79 \mathrm{~ns})<\alpha$-toluenethiol capped CdSe quantum dots $(11.49 \mathrm{~ns})<$ TOPO capped CdSe quantum dots (16.73 ns). The observed decrease in the PL intensity and emission lifetime is indicative of the fact that the ligand interaction with $\mathrm{CdSe}$ quantum dots results in charge transfer quenching. Thus, the sequence of the charge separation rates among the ligands is in the reverse order of their emission lifetimes.

To demonstrate that efficient hole transfer and charge transport are present in aromatic ligand capped $\mathrm{CdSe}$ quantum dots, we fabricated a photovoltaic device by spin-coating a solution of $2 \%$ by weight CdSe quantum dots onto an indium tin oxide glass substrate coated with PEDOT:PSS, then vacuum deposited an aluminium electrode (Fig. 7(a)). Three photovoltaic devices were fabricated using an active layer consisting of TOPO capped CdSe quantum dots, $\alpha$-toluenethiol capped CdSe quantum dots, and thiophenol capped CdSe quantum dots. Since the $p$-hydroxythiophenol ligand capped CdSe quantum dots were dissolved in ethanol that redissolved the PEDOT : PSS layer and caused the device to fail, we have no photovoltaic device results of $p$-hydroxythiophenol ligand capped CdSe quantum dots shown here. Fig. 7(b) shows a schematic energy level diagram for an ITO/PEDOT: PSS/CdSe/Al device, assuming that CdSe quantum dot acts as intrinsic materials. The ionization potential and electron affinity of CdSe quantum dots are estimated from the bulk values. ${ }^{64}$ As the electron-hole pairs are generated by incident light, electrons move toward the aluminium electrode and holes move toward the ITO electrode.

Fig. 7(c) shows the corresponding external quantum efficiency (EQE) of the CdSe quantum dot photovoltaic devices with different ligands. The EQE spectral response matches the lowest energy feature in the absorption spectrum for each sample. ${ }^{65}$ The device begins absorbing below $600 \mathrm{~nm}$, and the shape of the photocurrent spectral response follows the absorption spectrum. No photocurrent is observed for excitation below the band edge suggesting that optical excitation of charges directly out of subbandgap trap states makes a negligible contribution to the photocurrent. The spectral response is clear evidence that photogenerated carriers originate from electron-hole pairs created within individual quantum dots. The EQEs of thiophenol capped CdSe quantum dots, $\alpha$-toluenethiol capped CdSe quantum dots, and TOPO capped CdSe quantum dots at $560 \mathrm{~nm}$ are $11.6 \%$, $3.6 \%$, and $1.7 \%$ respectively, consistent with the charge transfer efficiency of thiophenol $>\alpha$-toluenethiol $>$ TOPO. The charge transport improves substantially to yield an enhancement by a factor of approximately 10, as the bulky ligand (TOPO) capped $\mathrm{CdSe}$ quantum dots were replaced by aromatic ligand (thiophenol) capped CdSe quantum dots. The low EQE found using TOPO capped CdSe quantum dots is primarily a consequence of (a)

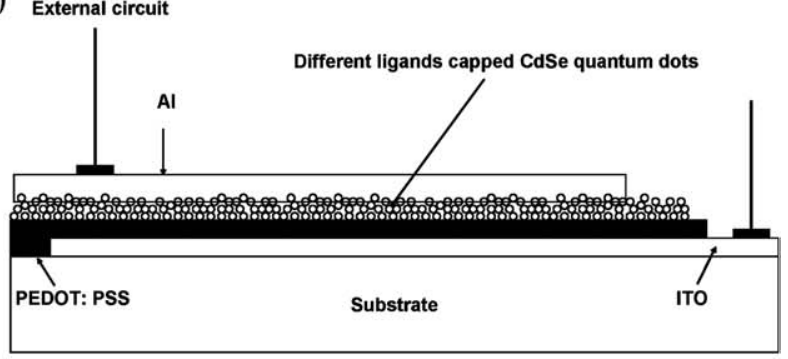

(b)

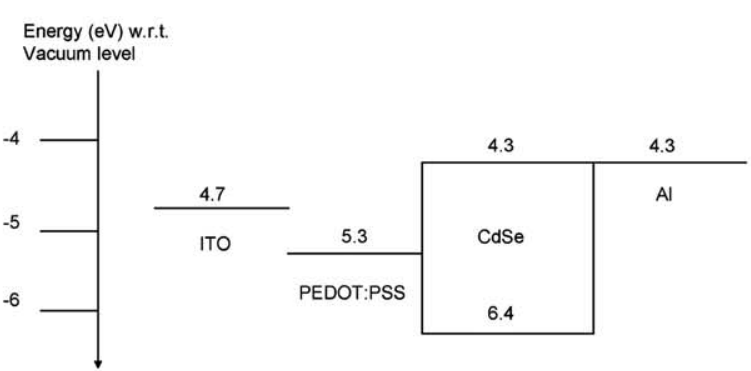

(c)

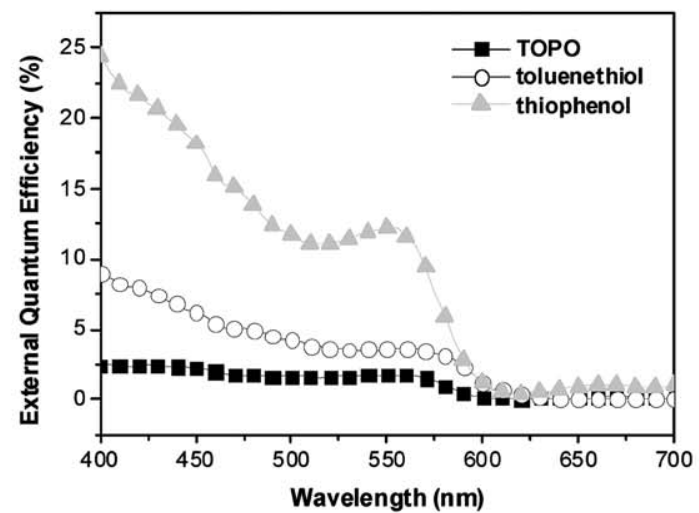

(d)

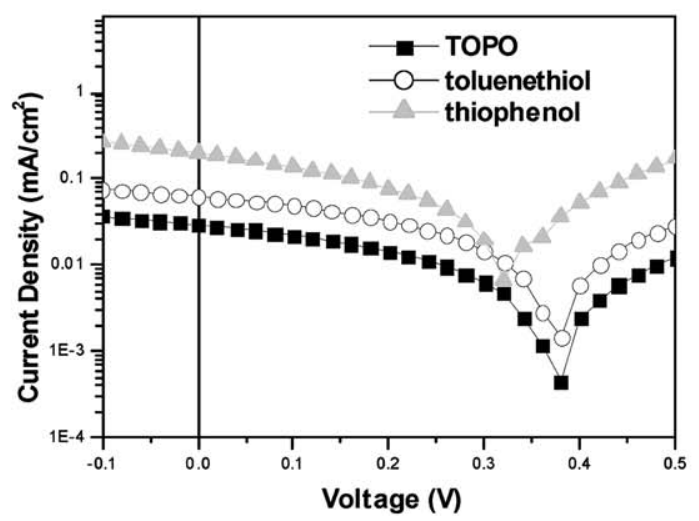

Fig. 7 (a) The device structure consists of an active layer of CdSe quantum dots with different ligands sandwiched between an aluminium electrode and a hole transport layer of PEDOT: PSS, which was deposited on an indium tin oxide glass substrate. (b) Energy level diagram for an ITO/PEDOT: PSS/CdSe/Al device. (c) External quantum efficiencies of CdSe quantum dots with different ligands. (d) The current-voltage characteristics of the devices containing CdSe quantum dots with different ligands under monochromatic illumination of $560 \mathrm{~nm}$ at $2.64 \mathrm{~mW} \mathrm{~cm}{ }^{-2}$. 
the fact that charge transfer is low compared to that in the aromatic ligand capped CdSe quantum dots. The TOPO barrier inhibits transport from quantum dot to quantum dot before recombination occurs. $I-V$ characteristics of fabricated devices were generated under monochromatic illumination of $560 \mathrm{~nm}$ (at the first exciting peak of CdSe quantum dots) at $2.64 \mathrm{~mW}$ $\mathrm{cm}^{-2}$, and shown in Fig. 7(d). The $I_{\mathrm{sc}}$ values of different ligands in increasing order are: thiophenol $>\alpha$-toluenethiol $>$ TOPO. These results are consistent with the lifetime and EQE data described above. $\pi$-Electrons in the thiophenol can facilitate efficient charge transport and charge separation, thus an order increase in $I_{\mathrm{sc}}$ was observed as compared with the $I_{\mathrm{sc}}$ of insulating TOPO. The efficiency of charge separation at the interface between thiophenol ligand and CdSe quantum dot can be further enhanced by a factor of 3 with respect to that in the $I_{\mathrm{sc}}$ of $\alpha$-toluenethiol. In the thiophenol capped CdSe quantum dot device, the fill factor is 0.26 , and the open-circuit voltage is approximately $0.32 \mathrm{~V}$, giving a power conversion efficiency of $0.62 \%$ at $2.64 \mathrm{~mW} \mathrm{~cm}^{-2}$. Although there are no conjugated polymers in our active layer, the short-circuit current density is comparable to literature results on organic bilayer or hybrid structure photovoltaic cells. ${ }^{66-69}$ This implies that our device made from thiophenol capped CdSe quantum dots exhibited the highest $I_{\mathrm{sc}}$ indicating efficient hole transfer and charge transport. However, the electron transport in the device is limited by the small size of the quantum dots. In principle, the efficiency of the device can be further optimized by increasing the size and changing the shape of the nanocrystals. Our different ligand capped CdSe quantum dots will be mixed with organic semiconductors as an active layer to study their photovoltaic properties in the future.

\section{Conclusion}

We have successfully synthesized aromatic ligand capped CdSe quantum dots through ligand exchange with TOPO capped CdSe quantum dots. The hole transfer behavior between colloidal CdSe quantum dots and ligands was studied by PL and time-resolved PL decay. We find that thiophenol and $p$-hydroxythiophenol effectively scavenge the photogenerated hole in CdSe, quenching quantum dot luminescence. The exciton lifetimes of both thiophenol and p-hydroxythiophenol are shorter than that of TOPO indicating the photogenerated holes in CdSe are effectively scavenged through the aromatic $\pi$-electrons. The exciton lifetime of $\alpha$-toluenethiol is shorter than that of TOPO, but larger than those of both thiophenol and $p$-hydroxythiophenol. Thus, the electron still can transfer from the aromatic ring to the CdSe quantum dots by tunneling through the methylene linkage between the aromatic ring and CdSe. Photovoltaic devices made of CdSe quantum dots with different ligands under monochromatic illumination at $560 \mathrm{~nm}$ (at the first exciting peak of CdSe quantum dots) at $2.64 \mathrm{~mW} \mathrm{~cm}{ }^{-2}$ show that the shortcircuit current density of thiophenol capped CdSe quantum dots is 10 times larger than that of TOPO capped CdSe quantum dots and about 3 times larger than that of $\alpha$-toluenethiol capped CdSe quantum dots, which indicates effective hole transport with aromatic ligands and quick charge separation at the interface of CdSe quantum dots. We have demonstrated that directly linking ligands to CdSe quantum dots will enhance their PL quenching and photoelectric properties.

\section{Acknowledgements}

This work was supported by the National Science Council of Republic of China (NSC95-2120-M-002-004) and US Air force (AOARD064-044). Mr An-Jey Su of University of Pittsburgh helped us to edit this manuscript.

\section{References}

1 M. Bruchez, M. Moronne, P. Gin, S. Weiss and A. P. Alivisatos, Science, 1998, 281, 2013-2018.

2 Y. Arima and H. Iwata, J. Mater. Chem., 2007, 17, 4079-4087.

3 N. Charvet, P. Reiss, A. Roget, A. Dupuis, D. Grunwald, S. Carayon, F. Chandezon and T. Livache, J. Mater. Chem., 2004, 14, 2638-2642.

4 N. C. Greenham, X. G. Peng and A. P. Alivisatos, Phys. Rev. B, 1996, 54, 17628-17637.

5 W. U. Huynh, X. G. Peng and A. P. Alivisatos, Adv. Mater., 1999, 11, 923-927.

6 W. U. Huynh, J. J. Dittmer and A. P. Alivisatos, Science, 2002, 295, 2425-2427.

7 B. Sun, E. Marx and N. C. Greenham, Nano Lett., 2003, 3, 961-963.

8 P. Wang, A. Abrusci, H. M. P. Wong, M. Svensson, M. R. Andersson and N. C. Greenham, Nano Lett., 2006, 6, 1789-1793.

9 Y. Y. Lin, C. W. Chen, J. Chang, T. Y. Lin, I. S. Liu and W. F. Su, Nanotechnology, 2006, 17, 1260-1263.

10 L. Han, D. Qin, X. Jiang, Y. Liu, L. Wang, J. Chen and Y. Cao, Nanotechnology, 2006, 17, 4736-4742.

11 A. V. Firth, Y. Tao, D. Wang, J. Ding and F. Bensebaa, J. Mater. Chem., 2005, 15, 4367-4372.

12 A. W. Tang, F. Teng, H. Jin, Y. H. Gao, Y. B. Hou, C. J. Liang and Y. S. Wang, Mater. Lett., 2007, 61, 2178-2181.

13 B. O. Dabbousi, M. G. Bawendi, O. Onitsuka and M. F. Rubner, Appl. Phys. Lett., 1995, 66, 1316-1318.

14 M. C. Schlamp, X. G. Peng and A. P. Alivisatos, J. Appl. Phys., 1997, 82, 5837-5842.

15 H. Mattoussi, L. H. Radzilowski, B. O. Dabbousi, E. L. Thomas, M. G. Bawendi and M. F. Rubner, J. Appl. Phys., 1998, 83, 79657974.

16 S. Coe, W. K. Woo, M. G. Bawendi and V. Bulovic, Nature, 2002, 420, 800-803.

17 M. Kuno, J. K. Lee, B. O. Dabbousi, F. V. Mikulec and M. G. Bawendi, J. Chem. Phys., 1997, 106, 9869-9882.

18 C. B. Murray, D. J. Norris and M. G. Bawendi, J. Am. Chem. Soc., 1993, 115, 8706-8715.

19 M. Q. Zhu, Em. Chang, J. Sun and R. A. Drezek, J. Mater. Chem., 2007, 17, 800-805.

20 Z. A. Peng and X. G. Peng, J. Am. Chem. Soc., 2001, 123, 183-184.

21 Z. A. Peng and X. G. Peng, J. Am. Chem. Soc., 2001, 123, 1389-1395.

22 S. D. Bunge, K. M. Krueger, T. J. Boyle, M. A. Rodriguez, T. J. Headley and V. L. Colvin, J. Mater. Chem., 2003, 13, 1705-1709.

23 P. Reiss, J. Bleuse and A. Pron, Nano Lett., 2002, 2, 781-784.

24 D. V. Talapin, A. L. Roagach, I. Mekis, S. Haubold, A. Kornowski, M. Haase and H. Weller, Colloids Surf., A, 2002, 202, 145-154.

25 M. Baumle, D. Stamou, J. M. Segura, R. Hovius and H. Vogel, Langmuir, 2004, 20, 3828-3831.

26 Y. Liu, M. Kim, Y. Wang, Y. A. Wang and X. G. Peng, Langmuir, 2006, 22, 6341-6345.

27 M. Green, P. Rahman and D. Smyth-Boyle, Chem. Commun., 2007, 574-576.

28 M. A. Hines and P. Guyot-Sionnest, J. Phys. Chem., 1996, 100, 468471.

29 X. G. Peng, T. E. Wilson, A. P. Alivisatos and P. G. Schultz, Angew. Chem., Int. Ed. Engl., 1997, 36, 145-147.

30 Y. A. Wang, J. J. Li, H. Y. Chen and X. G. Peng, J. Am. Chem. Soc., 2002, 124, 2293-2298.

31 H. Skaff and T. Emrick, Chem. Commun., 2003, 52-53.

32 D. J. Milliron, A. P. Alivisatos, C. Pitois, C. Edder and J. M. J. Frechet, Adv. Mater., 2003, 15, 58-61.

33 W. H. Binder, R. Sachsenhofer, C. J. Straif and R. Zirbs, J. Mater. Chem., 2007, 17, 2125-2132.

34 R. P. Andres, J. D. Bielefeld, J. I. Henderson, D. B. Janes, V. R. Kolagunta, C. P. Kubiak, W. J. Mahoney and R. G. Osifchin, Science, 1996, 273, 1690-1693. 
35 M. Pientka, V. Dyakonov, D. Meissner, A. Rogach, D. Talapin, H. Weller, L. Lutsen and D. Vanderzande, Nanotechnology, 2004, 15, 163-170.

36 O. Schmelz, A. Mews, T. Basche, A. Herrmann and K. Mullen, Langmuir, 2001, 17, 2861-2865.

37 M. Tomasulo, I. Yildiz, S. L. Kaanumalle and F. M. Raymo, Langmuir, 2006, 22, 10284-10290.

38 C. Bullen and P. Mulvaney, Langmuir, 2006, 22, 3007-3013.

39 J. Locklin, D. Patton, S. Deng, A. Baba, M. Millan and R. C. Advincula, Chem. Mater., 2004, 16, 5187-5193.

40 Q. Shang, H. Wang, H. Yu, G. Shan and R. Yan, Colloids Surf., A, 2007, 294, 86-91.

41 M. Y. Odoi, N. I. Hammer, K. Sill, T. Emrick and M. D. Barnes, J. Am. Chem. Soc., 2006, 128, 3506-3507.

42 M. Anni, L. Manna, R. Cingolani, D. Valerini, A. Creti and M. Lomascolo, Appl. Phys. Lett., 2004, 85, 4169-4171.

43 N. I. Hammer, K. T. Early, K. Sill, M. Y. Odoi, T. Emrick and M. D. Barnes, J. Phys. Chem. B, 2006, 110, 14167-14171.

44 C. Landes, C. Burda, M. Braun and M. A. El-Sayed, J. Phys. Chem. $B, 2001, \mathbf{1 0 5}, 2981-2986$.

45 B. R. Fisher, H. J. Eisler, N. E. Stott and M. G. Bawendi, J. Phys. Chem. B, 2004, 108, 143-148.

46 S. F. Wuister, C. M. Donega and A. Meijerink, J. Phys. Chem. B, 2004, 108, 17393-17397.

47 J. E. Bowen Katari, V. L. Colvin and A. P. Alivisatos, J. Phys. Chem., 1994, 98, 4109-4117.

48 A. Puzder, A. J. Williamson, N. Zaitseva, G. Galli, L. Manna and A. P. Alivisatos, Nano Lett., 2004, 4, 2361-2365.

49 A. Puzder, A. J. Williamson, F. Gygi and G. Galli, Phys. Rev. Lett., 2004, 92, 2174011-217404.

50 D. P. Masson, D. J. Lockwood and M. J. Graham, J. Appl. Phys., 1997, 82, 1632-1639.

51 G. Hollinger, G. Marest, H. Jaffrezic, J. Tousset and N. Moncoffge, Nucl. Instrum. Methods Phys. Res., Sect. B, 1985, 7/8, 177.

52 D. Briggs and M. P. Seah, Practical Surface Analysis, John Wiley and Sons, New York, 1993, vol. 1, second edition.
53 B. A. D. Angelis, C. Rizzo, S. Contarini and S. P. Howlett, Appl. Surf. Sci., 1991, 51, 177-183.

54 A. G. Young, D. P. Green and A. J. McQuillan, Langmuir, 2006, 22, 11106-11112.

55 B. Persson and B. Nygard, J. Electroanal. Chem. Interfac. Electrochem., 1974, 56, 373-383.

56 D. T. Sawyer, A. Sobkowiak, and J. L. Roberts, Electrochemistry for Chemists, 2nd edn, Wiley, NY, 1995, p. 192.

57 M. J. Natan, J. W. Thackeray and M. S. Wrighton, J. Phys. Chem., 1986, 90, 4089-4098.

58 M. G. Bawendi, P. J. Carroll, W. L. Wilson and L. E. Brus, J. Chem. Phys., 1992, 96, 946-954.

59 J. A. Kloepfer, S. E. Bradforth and J. L. Nadeau, J. Phys. Chem. B, 2005, 109, 9996-10003.

60 N. A. Hill and K. B. Whaley, J. Chem. Phys., 1994, 100, 2831-2837.

61 D. F. Underwood, T. Kippeny and S. J. Rosenthal, J. Phys. Chem. B, 2001, 105, 436-443.

62 K. Leung and K. B. Whaley, J. Chem. Phys., 1999, 110, 11012-11022.

63 K. Leung, S. Pokrant and K. B. Whaley, Phys. Rev. B, 1998, 57, 12291-12301.

64 T. C. Chiang, K. H. Frank, H. J. Freund, A. Goldmann, F. J. Himpsel, U. Karlsson, R. C. Leckey and W. D. Schneider, Electronic Structure of Solids, Landolt-Bornstein, New Series, Springer-Verlag, Berlin and Heidelberg, Group III, 1989, vol. 23.

65 E. J. D. K. Klem, D. D. MacNeil, P. W. Cyr, L. Levina and E. H. Sargent, Appl. Phys. Lett., 2007, 90, 1831131-1831133.

66 Z. Liang, K. L. Dzienis, J. Xu and Q. Wang, Adv. Funct. Mater., $2006,16,542-548$.

67 J. K. Mwaura, M. R. Pinto, D. Witker, N. Ananthakrishnan, K. S. Schanze and J. R. Reynolds, Langmuir, 2005, 21, 10119-10126.

68 H. Mattoussi, M. F. Rubner, F. Zhou, J. Kumar, S. K. Tripathy and L. Y. Chiang, Appl. Phys. Lett., 2000, 77, 1540-1542.

69 L. A. A. Pettersson, L. S. Roman and O. Inganas, J. Appl. Phys., 1999, 86, 487-496. 